\title{
Errata: Effect of face separation in corner-cube reflectors
}

Rohit Nair

Keith W. Goossen

University of Delaware

Department of Electrical and Computer Engineering

140 Evans Hall

Newark, Delaware 19716

E-mail: nair@ece.udel.edu

This article [Opt. Eng. 48(12), 123003 (December 2009)] was originally published on 22 December 2009 with a few minor errors.

1. In the caption to Fig. 1:

a. The word "retroreflected" was misspelled as "retroreected".

b. $\quad \hat{n}_{i}$, which is a unit vector, was missing the hat symbol (it was incorrectly written as $n_{i}$ ).

c. Finally, there should be a space between the word "simply" and " $-\hat{n}_{i}$ ".

For clarity, the complete correct caption to Fig. 1 is "Fig. 1 Schematic of a retroreflector (a) without and (b) with gaps between the faces. The angles $\theta$ and $\phi$ denote the direction, $\hat{n}_{i}$, of incidence of the interrogating ray and the $v$-axis in the optical coordinate system. The direction of the retroreflected ray, $\hat{n}_{o}$, is simply $-\hat{n}_{i}$. The three vectors $\hat{n}_{i}, \hat{n}_{o}$, and $\hat{v}$ are parallel to each other."

2. In the caption to Fig. 2, the variable $A_{R R \mid e f f}$ was incorrectly written as $A_{P R, e f f}$.

3. In the caption to Fig. 7, the variable $A_{R R \mid e f f_{o}}$ was incorrectly written as $A_{R R, e f f}$.

All versions of the article were corrected on 31 December 2009 and the article appears correctly in print. 